\title{
Signless Laplacian Energy of Operations on Intuitionistic Fuzzy Graphs
}

\author{
Obbu Ramesh ${ }^{1}$, S. Sharief Basha ${ }^{2}$ and W.Abdul Hameed ${ }^{3}$ \\ ${ }^{1}$ PhD Scholar, SAS, VIT University, Vellore 632 014, Tamilnadu, India; \\ ${ }^{2,3}$ SAS, VIT University, Vellore 632 014, Tamilnadu, India \\ *Corresponding author E-mail: rameshobbu@gmail.com
}

\begin{abstract}
After determining the Signless Laplacian energy of an Intuitionistic fuzzy graphs and the study of lower and upper boundaries of Signless Laplacian energy of an Intuitionistic fuzzy graphs, then we planned to search Signless Laplacian energy of an Intuitionistic fuzzy graphs on some operations such as Union and Join of the Intuitionistic Fuzzy Graphs and also examine the difference bet ween two Intuitionistic fuzzy graphs.
\end{abstract}

Keywords: Intuitionistic fuzzy graphs, Union operation, Join operation of two Intuitionistic fuzzy graphs.

\section{Introduction}

Fuzzy set has developed as a probable area of inter corrective study, and fuzzy graph theory is also of recent interest. The thought of a fuzzy graph relation was demarcated by Zadeh [6], and it has found solicitations in the analysis of cluster patterns. Rosenfeld [4] measured the fuzzy relations on fuzzy sets and developed the construction of fuzzy graphs.

In this paper we are disturbed with simple graphs. Let $G$ be a graph with $\mathrm{n}$ vertices and $\mathrm{m}$ edges, and we say this $\mathrm{G}$ is a $(n, m)$ graph. Let $\mathrm{d} i$ be the degree of $i$ th vertex of $\mathrm{G}, \mathrm{i}=1,2, \ldots, \mathrm{n}$. The spectrum of the graph $\mathrm{G}$, consisting of the numbers $\lambda_{1}, \lambda_{2}, \ldots, \lambda_{n}$, is the spectrum of its adjacency matrix [5]. The Laplacian spectrum of the graph $\mathrm{G}$, containing of the numbers $\mu_{1}, \mu_{2}, \ldots, \mu_{n}$, is the spectrum of its Laplacian matrix.

In this paper we introduce the concept of Signless Laplacian energy of operations on intuitionistic fuzzy graphs. Section 2 consists of different operations such as complement of an Intuitionistic fuzzy graph, union and join of two intuitionistic fuzzy graphs definition and we present the Signless Laplacian energy of union and join of two intuitionistic fuzzy graphs. We give the conclusion in the last section.

\section{Results}

Signless -Laplacian Energy of Some Operations on Intuitionistic Fuzzy Graphs

2.1 . Signless -Laplacian Energy of Complement of an Intuitionistic Fuzzy Graphs

Definition: - The complement of an Intuitionistic fuzzy graph

$\mathbf{G}=(\mathbf{V}, \mathbf{E})$ is an Intuitionistic fuzzy graph, $\bar{G}=(\bar{V}, \bar{E})$ where $\bar{V}=V \quad, \quad \mu_{1 i}=\mu_{i 1}$ and $\gamma_{1 i}=\gamma_{i 1}$ for all $\mathrm{i}=1,2, . ., \mathrm{n}$, $\bar{\mu}_{2 i j}=\min \left(\mu_{i 1}, \mu_{1 i}\right)-\mu_{2 i j}, \bar{\gamma}_{2 i j}=\min \left(\gamma_{i 1}, \gamma_{1 i}\right)-\gamma_{2 i j}$ for all $\mathrm{i}, \mathrm{j}=, 2, . ., \mathrm{n}$
Example 2.1.2:- Let $\mathrm{V}=\left\{v_{1}, v_{2}, v_{3}, v_{4}\right\}$

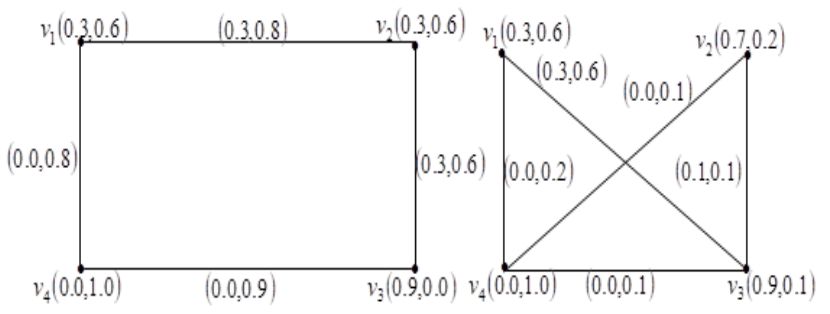

Fig. l: Intuitionistic fuzzy graph $G$ and $\tilde{G}$

The adjacency matrices of an intuitionistic fuzzy graph $G$ and complement of this intuitionistic fuzzy graph $\bar{G}$ are

$A(I F G)=\left[\begin{array}{cccc}0 & (0.3,0.8) & 0 & (0.0,0.8) \\ (0.3,0.8) & 0 & (0.6,0.1) & 0 \\ 0 & (0.6,0.1) & 0 & (0.0,0.9) \\ (0.0,1.0) & 0 & (0.0,0.9) & 0\end{array}\right]$

$A(I F \tilde{G})=\left[\begin{array}{cccc}0 & 0 & (0.3,0.6) & (0.0,0.2) \\ 0 & 0 & (0.1,0.1) & (0.0,1.0) \\ (0.3,0.6) & (0.1,0.1) & 0 & (0.0,0.1) \\ (0.0,0.2) & (0.0,1.0) & (0.0,0.1) & 0\end{array}\right]$

$A\left(\mu_{i j}\right)=\left[\begin{array}{cccc}0 & 0.3 & 0 & 0 \\ 0.3 & 0 & 0.6 & 0 \\ 0 & 0.6 & 0 & 0 \\ 0 & 0 & 0 & 0\end{array}\right]$ 


$$
A\left(\gamma_{i j}\right)=\left[\begin{array}{cccc}
0 & 0.8 & 0 & 0.8 \\
0.8 & 0 & 0.1 & 0 \\
0 & 0.1 & 0 & 0.9 \\
1.0 & 0 & 0.9 & 0
\end{array}\right]
$$

Signless Laplacian matrix of the membership and non- membership value of an intuitionistic fuzzy graph $\mathrm{G}$ are

$$
\begin{aligned}
Q\left(\mu_{i j}\right) & =\left[\begin{array}{cccc}
0.3 & 0.3 & 0 & 0 \\
0.3 & 0.9 & 0.6 & 0 \\
0 & 0.6 & 0.6 & 0 \\
0 & 0 & 0 & 0
\end{array}\right] \\
Q\left(\gamma_{i j}\right) & =\left[\begin{array}{cccc}
1.6 & 0.8 & 0 & 0.8 \\
0.8 & 0.9 & 0.1 & 0 \\
0 & 0.1 & 1.0 & 0.9 \\
1.0 & 0 & 0.9 & 1.7
\end{array}\right]
\end{aligned}
$$$$
\operatorname{spec}\left(Q\left(\mu_{i j}(I F G)\right)\right)=\{-0.0,0,0.3804,1.4196\}
$$$$
\operatorname{spec}\left(Q\left(\gamma_{i j}(I F G)\right)\right)=\{3.0147,1.7060,0,0.6794\}
$$$$
S L E\left(\mu_{i j}(I F G)\right)=\left|0-\frac{2(1.8)}{4}\right|+\left|0-\frac{2(1.8)}{4}\right|+\left|0.3804-\frac{2(1.8)}{4}\right|+\left|1.4196-\frac{2(1.8)}{4}\right|
$$

$$
=1.9392
$$

$\operatorname{SLE}\left(\gamma_{i j}(I F G)\right)=\left|3.0147-\frac{2(5.4)}{4}\right|+\left|1.7060-\frac{2(5.4)}{4}\right|+\left|0-\frac{2(5.4)}{4}\right|+\left|0.6794-\frac{2(5.4)}{4}\right|$ $=4.0412$

The Signless laplacian energy of complement of an intuitionistic fuzzy graph $\tilde{G}$ are

$$
\begin{aligned}
A\left(\tilde{\mu}_{i j}\right) & {\left[\begin{array}{cccc}
0 & 0 & 0.3 & 0 \\
0 & 0 & 0.1 & 0 \\
0.3 & 0.1 & 0 & 0 \\
0 & 0 & 0 & 0
\end{array}\right] } \\
A\left(\tilde{\gamma}_{i j}\right) & =\left[\begin{array}{cccc}
0 & 0 & 0.6 & 0.2 \\
0 & 0 & 0.1 & 1.0 \\
0.6 & 0.1 & 0 & 0.1 \\
0.2 & 1.0 & 0.1 & 0
\end{array}\right]
\end{aligned}
$$

Signless Laplacian matrix of the membership and non membership value of a complement of intuitionistic fuzzy graph $\tilde{G}$ are

$$
\begin{aligned}
Q\left(\tilde{\mu}_{i j}\right) & =\left[\begin{array}{cccc}
0.3 & 0 & 0.3 & 0 \\
0 & 0.1 & 0.1 & 0 \\
0.3 & 0.1 & 0.4 & 0 \\
0 & 0 & 0 & 0
\end{array}\right] \\
Q\left(\tilde{\gamma}_{i j}\right) & =\left[\begin{array}{cccc}
0.8 & 0 & 0.6 & 0.2 \\
0 & 1.1 & 0.1 & 1.0 \\
0.6 & 0.1 & .0 .8 & 0.1 \\
0.2 & 1.0 & 0.1 & 1.3
\end{array}\right]
\end{aligned}
$$

$\operatorname{spec}\left(Q\left(\mu_{i j}(I F \tilde{G})\right)\right)=\{-0.0,0,0.1354,0.6646\}$

$\operatorname{spec}\left(Q\left(\gamma_{i j}(I F \tilde{G})\right)\right)=\{0.0943,0.2939,1.3575,2.2543\}$

$\operatorname{SLE}\left(\tilde{\gamma}_{i j}(I F \tilde{G})\right)=\left|0.0943-\frac{2(2)}{4}\right|+\left|0.2939-\frac{2(2)}{4}\right|+\left|1.3575-\frac{2(2)}{4}\right|+\left|2.2543-\frac{2(2)}{4}\right|$

$=3.2237$

2.2: - Signless Laplacian energy of Union of two intuitionistic fuzzy graphs:

\section{Definition 2.2.1 :-Union of intuitionistic fuzzy graph}

Let $G_{1}=\left(V_{1}, E_{1}\right)$ and $G_{2}=\left(V_{2}, E_{2}\right)$ be any two intuition-

istic fuzzy graphs with $V_{1} \cap V_{2}=\phi \quad$ and $G=G_{1} \cap G_{2}=\left(V_{1} \cap V_{2}, E_{1} \cap E_{2}\right)$. Now the union of intuitionistic fuzzy graphs $G_{1}$ and $G_{2}$ is also an intuitionistic fuzzy graph defined by

$\left(\mu_{1} \cup \mu_{1}^{\prime}\right)(v)=\left\{\begin{array}{l}\mu_{1}(v) \text { if } v \in V_{1}-V_{2} \\ \mu_{1}^{\prime}(v) \text { if } v \in V_{2}-V_{1}\end{array}\right.$
$\left(\gamma_{1} \cup \gamma_{1}^{\prime}\right)(v)=\left\{\begin{array}{l}\gamma_{1}(v) \text { if } v \in V_{1}-V_{2} \\ \gamma_{1}^{\prime}(v) \text { if } v \in V_{2}-V_{1}\end{array}\right.$
$\left(\mu_{1} \cup \mu_{1}^{\prime}\right)\left(v_{i} v_{j}\right)=\left\{\begin{array}{l}\mu_{2 i} \text { if } e_{i v} \in E_{1}-E_{2} \\ \mu_{2 i}^{t} \text { if } e_{i v} \in E_{2}-E_{1}\end{array}\right.$

$\left(\gamma_{1} \cup \gamma_{1}^{\prime}\right)\left(v_{i} v\right)=\left\{\begin{array}{l}\gamma_{2 \psi} \text { if } e_{i v} \in E_{1}-E_{2} \\ \gamma_{2 \psi}^{\prime} \text { if } e_{i j} \in E_{2}-E_{1}\end{array}\right.$

Where $\left(\mu_{1}, \gamma_{1}\right)$ and $\left(\mu_{1}^{\prime}, \gamma_{1}^{\prime}\right)$ refer the vertex membership and non-membership of $G_{1}$ and $G_{2}$ respectively; $\left(\mu_{2}, \gamma_{2}\right)$ and $\left(\mu_{2}^{\prime}, \gamma_{2}^{\prime}\right)$ refer the edge membership and non- membership of $G_{1}$ and $G_{2}$ respectively;
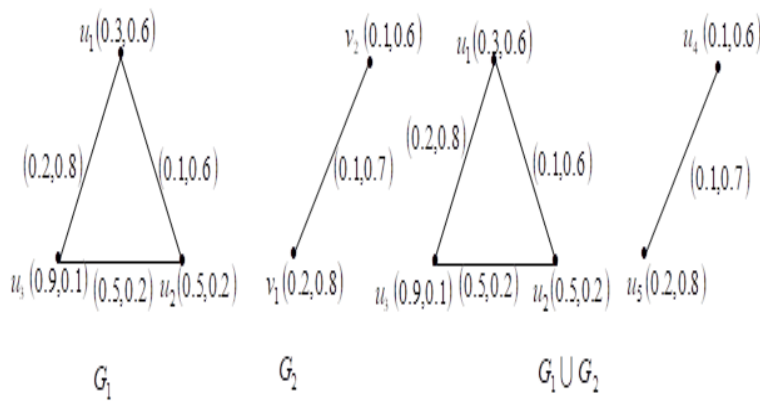

\section{Fig.2: Intuitionistic Fuzzy Graphs $G_{1}, G_{2}$ and $G_{1} \cup G_{2}$}

Adjacency matrices of $G_{1}$ and $G_{2}$ are given below

$$
\begin{aligned}
& A\left(G_{1}\right)=\left[\begin{array}{ccc}
0 & (0.1,0.6) & (0.2,0.8) \\
(0.1,0.6) & 0 & (0.5,0.2) \\
(0.2,0.8) & (0.5,0.2) & 0
\end{array}\right] \\
& A\left(G_{2}\right)=\left[\begin{array}{cc}
0 & (0.1,0.7) \\
(0.1,0.7) & 0
\end{array}\right]
\end{aligned}
$$

$\operatorname{SLE}\left(\tilde{\mu}_{i j}(I F \tilde{G})\right)=\left|-0.0-\frac{2(0.4)}{4}\right|+\left|0-\frac{2(0.4)}{4}\right|+\left|0 . .1354-\frac{2(0.4)}{4}\right|+\left|0.6646-\frac{2(0.4)}{4}\right|$ The membership and non- membership values of $G_{1}$ 


$$
\begin{aligned}
& A\left(\mu_{i j}\left(G_{1}\right)\right)=\left[\begin{array}{ccc}
0 & 0.1 & 0.2 \\
0.1 & 0 & 0.5 \\
0.2 & 0.5 & 0
\end{array}\right] \\
& A\left(\gamma_{i j}\left(G_{1}\right)\right)=\left[\begin{array}{ccc}
0 & 0.6 & 0.8 \\
0.6 & 0 & 0.5 \\
0.8 & 0.2 & 0
\end{array}\right] \\
& \text { and } A\left[\mu_{i j}\left(G_{2}\right)\right]=\left[\begin{array}{cc}
0 & 0.1 \\
0.1 & 0
\end{array}\right] \\
& A\left[\gamma_{i j}\left(G_{2}\right)\right]=\left[\begin{array}{ccc}
0 & 0.7 \\
0.7 & 0
\end{array}\right] \\
& Q\left[\mu_{i j}\left(G_{1}\right)\right]=\left[\begin{array}{ccc}
0.3 & 0.1 & 0.2 \\
0.1 & 0.6 & 0.5 \\
0.2 & 0.5 & 0.7
\end{array}\right] \\
& Q\left[\gamma_{i j}\left(G_{1}\right)\right]=\left[\begin{array}{ccc}
1.4 & 0.6 & 0.8 \\
0.6 & 0.8 & 0.5 \\
0.8 & 0.2 & 1.0
\end{array}\right] \\
& \operatorname{spec}\left[Q\left(\mu_{i j}\left(G_{1}\right)\right)\right]=\{0.1207,0.2753,1.2040\} \\
& \operatorname{spec}\left[Q\left(\gamma_{i j}\left(G_{1}\right)\right)\right]=\{2.4247,0.4330,0.6393\} \\
& \operatorname{sLE}\left[\mu_{i j}\left(G_{1}\right)\right]=\left|0.1207-\frac{2(0.8)}{3}\right|+\left|0.2753-\frac{2(0.8)}{3}\right|+\left|1.2040-\frac{2(0.8)}{3}\right| \\
& =1.3413 \\
& \text { SLE }\left[\gamma_{i j}\left(G_{1}\right)\right]=\left|2.4277-\frac{2(1.75)}{3}\right|+\left|0.4330-\frac{2(1.75)}{3}\right|+\left|0.6393-\frac{2(1.75) \mid}{3}\right| \\
& =2.5221
\end{aligned}
$$

Similarly the membership and non -membership values of $G_{2}$

$$
\begin{aligned}
& A\left(\mu_{i j}\left(G_{2}\right)\right)=\left[\begin{array}{cc}
0 & 0.1 \\
0.1 & 0
\end{array}\right] \text { and } \\
& A\left(\gamma_{i j}\left(G_{2}\right)\right)=\left[\begin{array}{cc}
0 & 0.7 \\
0.7 & 0
\end{array}\right] \\
& Q\left(\mu_{i j}\left(G_{2}\right)\right)=\left[\begin{array}{ll}
0.1 & 0.1 \\
0.1 & 0,1
\end{array}\right] \text { and } \\
& Q\left(\gamma_{i j}\left(G_{2}\right)\right)=\left[\begin{array}{ll}
0.7 & 0.7 \\
0.7 & 0,7
\end{array}\right] \\
& \operatorname{Spec}\left[Q\left(\mu_{i j}\left(G_{2}\right)\right)\right]=\{0,0.2\} \\
& \operatorname{Spec}\left[Q\left(\gamma_{i j}\left(G_{2}\right)\right)\right]=\{0,1.4\} \\
& \operatorname{SLE}\left[\mu_{i j}\left(G_{2}\right)\right]=\left|0-\frac{2(0.1)}{2}\right|+\left|0.2-\frac{2(0.1)}{2}\right|=0.1+0.1=0.2 \\
& \operatorname{SLE}\left[\gamma_{i j}\left(G_{2}\right)\right]=\left|0-\frac{2(0.7)}{2}\right|+\left|1.4-\frac{2(0.7)}{2}\right|=0.7+0.7=1.4
\end{aligned}
$$

Adjacency matrix of $G_{1} \cup G_{2}$ is given below

$$
A\left[G_{1} \cup G_{2}\right]=\left[\begin{array}{ccccc}
0 & (0.1,0.6) & (0.2,0.8) & 0 & 0 \\
(0.1,0.6) & 0 & (0.5,0.2) & 0 & 0 \\
(0.2,0.8) & (0.5,0.2) & 0 & 0 & 0 \\
0 & 0 & 0 & 0 & (0.1,0.7) \\
0 & 0 & 0 & (0.1,0.7) & 0
\end{array}\right]
$$

The membership matrix of $G_{1} \cup G_{2}$ is

$$
\begin{aligned}
& \mu_{i j}\left[G_{1} \cup G_{2}\right]=\left[\begin{array}{ccccc}
0 & 0.1 & 0.2 & 0 & 0 \\
0.1 & 0 & 0.5 & 0 & 0 \\
0.2 & 0.5 & 0 & 0 & 0 \\
0 & 0 & 0 & 0 & 0.1 \\
0 & 0 & 0 & 0.1 & 0
\end{array}\right] \\
& Q\left[\mu_{i j}\left[G_{1} \cup G_{2}\right]\right]=\left[\begin{array}{ccccc}
0.3 & 0.1 & 0.2 & 0 & 0 \\
0.1 & 0.6 & 0.5 & 0 & 0 \\
0.2 & 0.5 & 0.7 & 0 & 0 \\
0 & 0 & 0 & 0.1 & 0.1 \\
0 & 0 & 0 & -0.1 & 0.1
\end{array}\right]
\end{aligned}
$$

$\operatorname{Spec}\left[Q\left(\mu_{i j}\left(G_{1} \cup G_{2}\right)\right)\right]=\{0,0.1207,0.2,0.2753,1.2040\}$ $\begin{aligned} S L E\left[\mu_{i j}\left(G_{1} \cup G_{2}\right)\right] & =\left|0-\frac{2(0.9)}{5}\right|+0.1207-\frac{2(0.9)}{5}|+| 0.2-\frac{2(0.9)}{5}|+| 0.2753-\frac{2(0.9)}{5}|+| 1.2040-\frac{2(0.9)}{5} \mid \\ & =1.6880\end{aligned}$

$\gamma_{i j}\left[G_{1} \cup G_{2}\right]=\left[\begin{array}{ccccc}0 & 0.6 & 0.8 & 0 & 0 \\ 0.6 & 0 & 0.2 & 0 & 0 \\ 0.8 & 0.2 & 0 & 0 & 0 \\ 0 & 0 & 0 & 0 & 0.7 \\ 0 & 0 & 0 & 0.7 & 0\end{array}\right]$

$Q\left[\mu_{i j}\left[G_{1} \cup G_{2}\right]\right]=\left[\begin{array}{ccccc}1.4 & 0.6 & 0.8 & 0 & 0 \\ 0.6 & 0.8 & 0.2 & 0 & 0 \\ 0.8 & 0.2 & 1.0 & 0 & 0 \\ 0 & 0 & 0 & 0.7 & 0.7 \\ 0 & 0 & 0 & 0.7 & 0.7\end{array}\right]$

$\operatorname{Spec}\left[Q\left(\gamma_{i j}\left(G_{1} \cup G_{2}\right)\right)\right]=\{0,0.2481,0.6817,1.4,2.2702\}$

$$
\begin{aligned}
S L E\left[\gamma_{i j}\left(G_{1} \cup G_{2}\right)\right] & =\left|0-\frac{2(2.3)}{5}\right|+\left|0.2481-\frac{2(2.3)}{5}\right|+ \\
\left|0.6817-\frac{2(2.3)}{5}\right|+\left|1.4-\frac{2(2.3)}{5}\right|+\left|2.2702-\frac{2(2.3)}{5}\right| & =3.6604
\end{aligned}
$$

We observed here that

$S L E\left[\mu_{i j}\left(G_{1}\right)\right]+S L E\left[\mu_{i j}\left(G_{2}\right)\right] \leq S L E\left[\mu_{i j}\left(G_{1} \cup G_{2}\right)\right]$ and also

$S L E\left[\gamma_{i j}\left(G_{1}\right)\right]+S L E\left[\gamma_{i j}\left(G_{2}\right)\right] \leq S L E\left[\gamma_{i j}\left(G_{1} \cup G_{2}\right)\right]$

2.3: - Signless Laplacian energy of join of two intuitionistic fuzzy graphs:

Definition 2.3.1:- The join of two intuitionistic fuzzy graph's $G_{1}$ and $G_{2}$ is an intuitionistic fuzzy graph

$G_{1}+G_{2}-\left(V_{1} \cup V_{2}, E_{1} \cup E_{2}\right)$ defined by

$\left(\mu_{1}+\mu_{1}^{\prime}\right)(v)=\left(\mu_{1} \cup \mu_{1}^{\prime}\right)(v)$ if $v \in V_{1} \cup V_{2}$

$\left(\gamma_{1}+\gamma_{1}^{\prime}\right)(v)=\left(\gamma_{1} \cup \gamma_{1}^{\prime}\right)(v)$ if $v \in V_{1} \cup V_{2}$

$\left(\mu_{2}+\mu_{2}^{\prime}\right)\left(v_{i} v_{j}\right)=\left(\mu_{2} \cup \mu_{2}^{\prime}\right)\left(v_{i} v_{j}\right)$ if $v_{i} v_{j} \in E_{1} \cup E_{2}$ and $=\min \left(\mu_{1}\left(v_{i}\right), \mu_{1}^{\prime}\left(v_{j}\right)\right)$ if $v_{i} v_{j} \in E^{\prime}$ $\left(\gamma_{2}+\gamma_{2}^{\prime}\right)\left(v_{i} v_{j}\right)=\left(\gamma_{2} \cup \gamma_{2}^{\prime}\right)\left(v_{i} v_{j}\right)$ if $v_{i} v_{j} \in E_{1} \cup E_{2}$ $=\max \left(v_{1}\left(v_{i}\right), \mu_{1}^{\prime}\left(v_{j}\right)\right)$ if $v_{i} v_{j} \in E^{\prime}$

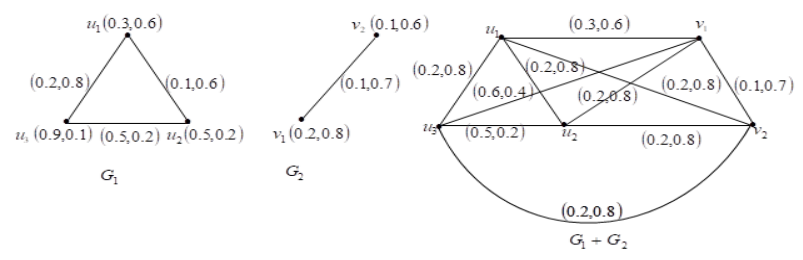


In the previous section we already find the

$\operatorname{SLE}\left(G_{1}\right)=(1.3413,2.5221)$

$\operatorname{SLE}\left(G_{2}\right)=(0.2,1.4)$

Now we will verify the laplacian energy of join of two intuitionistic fuzzy graph $G=G_{1}+G_{2}$

$A\left(G_{1}+G_{2}\right)=\left[\begin{array}{ccccc}0 & (0.1,0.6) & (0.2,0.8) & (0.3,0.6) & (0.2,0.8) \\ (0.1,0.6) & 0 & (0.5,0.0) & (0.2,0.8) & (0.2,0.8) \\ (0.2,0.8) & (0.5,0.0) & 0 & (0.6,0.4) & (0.2,0.8) \\ (0.3,0.6) & (0.2,0.8) & (0.6,0.4) & 0 & (0.1,0.7) \\ (0.2,0.8) & (0.2,0.8) & (0.2,0.8) & (0.1,0.7) & 0\end{array}\right]$

$\begin{aligned} & A\left[\mu_{i j}\left(\left(G_{1}+G_{2}\right)\right)\right]= {\left[\begin{array}{ccccc}0 & 0.1 & 0.2 & 0.3 & 0.2 \\ 0.1 & 0 & 0.5 & 0.2 & 0.2 \\ 0.2 & 0.5 & 0 & 0.6 & 0.2 \\ 0.3 & 0.2 & 0.6 & 0 & 0.1 \\ 0.2 & 0.2 & 0.2 & 0.1 & 0\end{array}\right] } \\ & Q\left[\mu_{i j}\left(\left(G_{1}+G_{2}\right)\right)\right]=\left[\begin{array}{ccccc}0.8 & 0.1 & 0.2 & 0.3 & 0.2 \\ 0.1 & 1.0 & 0.5 & 0.2 & 0.2 \\ 0.2 & 0.5 & 1.5 & 0.6 & 0.2 \\ 0.3 & 0.2 & 0.6 & 1.2 & 0.1 \\ 0.2 & 0.2 & 0.2 & 0.1 & 0.7\end{array}\right]\end{aligned}$

$\operatorname{Spec}\left[Q\left(\mu_{i j}\left(G_{1}+G_{2}\right)\right)\right]=\{0.4883,0.5910,0.8259,0.9537,2.3411\}$

$S L E\left[\mu_{i j}\left(G_{1}+G_{2}\right)\right]=\left|0.4883-\frac{2(2.6)}{5}\right|+\left|0.5910-\frac{2(2.6)}{5}\right|+\left|0.8259-\frac{2(2.6)}{5}\right|+$

$\left|0.9537-\frac{2(2.6)}{5}\right|+\left|2.3411-\frac{2(2.6)}{5}\right|$

$=2.6021$

$A\left[\gamma_{i j}\left(\left(G_{1}+G_{2}\right)\right)\right]=\left[\begin{array}{ccccc}0 & 0.6 & 0.8 & 0.6 & 0.8 \\ 0.6 & 0 & 0 & 0.8 & 0.8 \\ 0.8 & 0 & 0 & 0.4 & 0.8 \\ 0.6 & 0.8 & 0.4 & 0 & 0.7 \\ 0.8 & 0.8 & 0.8 & 0.7 & 0\end{array}\right]$

$Q\left[\gamma_{i j}\left(\left(G_{1}+G_{2}\right)\right)\right]=\left[\begin{array}{ccccc}2.8 & 0.6 & 0.8 & 0.6 & 0.8 \\ 0.6 & 2.2 & 0 & 0.8 & 0.8 \\ 0.8 & 0 & 2.0 & 0.4 & 0.8 \\ 0.6 & 0.8 & 0.4 & 2.5 & 0.7 \\ 0.8 & 0.8 & 0.8 & 0.7 & 3.1\end{array}\right]$

$\operatorname{Spec}\left[Q\left(\gamma_{i j}\left(G_{1}+G_{2}\right)\right)\right]=\{1.1361,1.7443,2.1315,2.3775,5.2107\}$

$S L E\left[\gamma_{i j}\left(G_{1}+G_{2}\right)\right]=\left|1.1361-\frac{2(6.3)}{5}\right|+\left|1.7443-\frac{2(6.3)}{5}\right|+$

$\left|2.1315-\frac{2(6.3)}{5}\right|+\left|2.3775-\frac{2(6.3)}{5}\right|+\left|5.2107-\frac{2(6.3)}{5}\right|$

$=5.3814$

We observed here that

$S L E\left[\mu_{i j}\left(G_{1}\right)\right]+S L E\left[\mu_{i j}\left(G_{2}\right)\right] \leq S L E\left[\mu_{i j}\left(G_{1}+G_{2}\right)\right]$ and

also

$S L E\left[\gamma_{i j}\left(G_{1}\right)\right]+S L E\left[\gamma_{i j}\left(G_{2}\right)\right] \leq S L E\left[\gamma_{i j}\left(G_{1}+G_{2}\right)\right]$

\section{Conclusion}

The Signless Laplacian matrix and energy for an intuitionistic fuzzy graph are defined. Some results on Laplacian spectra of intuitionistic fuzzy graphs may reveal more analogous results of these kinds and will be discussed in the forthcoming papers.

\section{Acknowledgement}

This is a text of acknowledgements. Do not forget people who have assisted you on your work. Do not exaggerate with thanks. If your work has been paid by a Grant, mention the Grant name and number here.

\section{References}

[1] Atanassov.K, Intuitionistic fuzzy sets, Fuzzy sets and systems, (20)87-96, (1986).

[2] Atanassov, K.: Intuitionistic Fuzzy sets : Theory and Applications, Springer-Verlag,Heidelberg, (1999).

[3] Gutman,I.: Gutman,I. The Energy of a Graph, Ber. Math - Statist. Sekt. Forschungsz. Graz, (103)1-22, (1978).

[4] Rosenfeld.A, Fuzzy Graphs, in Zadeh.L.A, Fu. K.cS, Tanaka.K and Shimura.M (eds.), Fuzzy Sets and their

[5] Applications to Cognitive and Decision Process, Academic Press, New York, 75-95, (1975).

[6] Sharief Basha.S and E. Kartheek, Laplacian Energy of an Intuitionistic Fuzzy Graph, Indian Journal of Science and Technology, 8(33) 1-7, (2015).

[7] Zadeh.L.A., Fuzzy sets , Information and Control,, (8)338-353, (1965). 